
PROTECTIVE FACTORS DEINITIONS: VULNERABILITY AND PROTECTIVE FACTOR

ASSOCIATED WITH CHILDHOOD ABUSE EXPOSURE

Although researchers have identified numerous correlates of child maltreatment, many studies frame these variables as predictors of child mat maltreatme treatment. However, as Stevens a contribute to child abuse in idenmight understand the various factors that contribute to child abuse fercemilies, tified patterns, maltreatment often occurs in multiple systins (ear ways that are neighborhoods, societies) that interact in complex and nonlinear ways thare ne not entirely predictable. In addied that there are no identifiable factors that studies, Finkelhor (1993) concluded that there are can absolutely protect a child from being sexually abused. We extend these ideas to propose that there are no markers-at the individual, familial, or societal level - to definitively protect a child from or predict the experince of that type of abuse or neglect. However, we can identify vulnerability factors that type of abuse or nized to be associated with higher rates of maltreatment. For his children iddere against exposure; we also include their peers, certain factors can protect

discussions of these protective factors.

Although child abuse research has advanced rapidly in recent decades to include more complex analyses and rigorous study designs, progress halize at times been complicated by variations in how researchers operationat child abuse across studies. Although researchers might uniformly agr that a 10-year-old child intentionally struck with enough force to cause broken bones has been physically abused, researchers in diffent broad definitions in counting spanking as physical abuse. Details within broates hildhood incer comple, the age that separates childhood are not always consed abuse differs widely, with cutoffs ranging from 14 to abuse from adulthood abuse differs widely, Inclusions of specific acts of 18 years (see Senn, Carey, \& Vanable, 2008). Inclusions of specific acture, abuse also vary from study to study, with some studies including exposure, threats, and attempts and others focusing only on more seve abuse and/or abuse. Some methodologies rely on substantiated records of abuse and/or neglect to identify abuse, whereas others rely on retrospective reporting from parents or other adult caregivers. While these methodological differences are to be expected in a relatively new area of research, arric pursuit. Although vergence will serve to improve the rigor of our scientific pursuit. Althoug this issue remains to be addressed by the field, for the purposes of this chapter, we include a broad range of definitions to ensure a comprehensive review of findings. Unless otherwise specified, the terms child maltreatment and child abuse refer to childhood physical, sexual, psychological, and emotional abuse; witnessing violence and neglect will be referred specifically when

CHU ET AL. maltreatment, neglect, and witnessing violence that occurred before the victim's 19 th birthday.

\section{WHAT DO WE KNOW?}

In this section, we review individual, parental and familial, and environmental and social network characteristics that appear to contribute to child abuse rates. A better understanding of the risk and protective factors that contribute to childhood maltreatment may guide researchers and service providers seeking to prevent future abuse.

\section{Individual Factors}

Several individual factors have emerged as potential moderators of child abuse exposure, including gender, age, and disability status. Gender appears to be a risk factor for child abuse and neglect generally. Child abuse and neglect cases reported to child protective service agencies across the nation are more likely to involve girls than boys (U.S. Department of Health and Human Services [USDHHS], 2008). However, researchers asking adult victims for retrospective reports using specific behavioral items have demonstrated striking differences in occurrence rates within abuse types and abuse characteristics by gender (e.g., Edinburgh, Saewyc, Thao, \& Levitt, 2006; Goldberg $\&$ Freyd, 2006). For example, girls tend to report child sexual abuse (CSA) exposure more than boys, whether interviewed as children (as reviewed in Holmes \& Slap, 1998) or in adulthood (Finkelhor, Hotaling, Lewis, \& Smith, 1990; Goldberg \& Freyd, 2006). A review of epidemiological \&tudies indicates that girls are at about 2.5 to 3 times higher risk than boys of sexual abuse (Finkelhor, 1993). Researchers have also found that more adult women than adult men retrospectively reported psychological or emotional maltreatment in childhood (Goldberg \& Freyd, 2006). In contrast, a review of nationally representative samples found no gender differences in relation to child physical abuse (Black, Heyman, \& Slep, 2001).

The relationship between perpetrator and victim also varies based on the victim's gender. For example, adult women tended to report victimization events in childhood perpetrated by someone close, whereas adult men tended to report more childhood victimization events perpetrated by someone not close to them (Goldberg \& Freyd, 2006). Perpetrators against boys were more likely to be closer in age to the victims, whereas perpetrators against girls have been reported to be older in age (Edinburgh et al., 2006). Thus, not only does gender moderate prevalence rates of broad categories of abuse, but gender effects also emerge when examining more specific relationships regarding abuse characteristics. 
Findings on gender and risk are consistent with betrayal trauma theory 1 will be associated with alterations in abuse, and proposes that such abuse will be associated with a Prince, \& typical information processing (DePrince \& Freyd, 2002, Freyd, DePrince, Gleaves, 2007). To the extent that girls are more likely to experience abuse 列 by close others on whom they are dependent, they are experience alterations in cognitive, emotional, and social inforis cessing than their male peers. These alterations, in turn, may increase girls' risk of exposure to additional victimizations (e.g., dating, IPV) as well as negative consequences of abuse (e.g., posttraumatic stress disorder).

Rence argued that Rese lack of underlie the gender asymo \& Luca, 2001). attention given to studying male sexual abuse (Romano \& De Luca, 2001 ) Until the mid-1980s, studies specifically examining male sexual victimization rarely took place. Although researchers have increasingly started focusing on male sexual abuse since then, the study of sexually abused females continue male sexual abuse since then, the stes in research studies. It is interesting that to garner more attention than males in research studies. ims in relive to female the media seem to pay particular attention to male victims relative to female victims, as is evidenced by the coverage of male victims in priest abus (1ctims. Furthermore, many people assume males may compared with fernale victim. be more reluctant than females to disclose abuse, althogh port for this commonly held belief is mixed (see Tang, Freyd, $\alpha$ Wang, 2008). A number factors may be involved in males' reluctance to disclose sexua A number of factors male ethics of abuse compared with females, including stuggling self-reliance, stigma of homosexuality, and less likelihood to label certain childhood sexual activities with another individual as abusive (for reviews, see ches factors may contribute to underestimates of male exposure and less understanding of the chales may and outcomes associated with male sexual abuse. Alternatively, females may be socialized to perceive (and report) violations in close relationships malso so than males (DePrince \& Freyd, 2002). Mental health professionals also continue to be biased against the existence of male sexual abuse, thus, profescontinue to leal with child sionals rarely ask adult males systematic investigation male victims (Romano \& De Luca, 2001). Because systems between sexually of male sexual abuse continues to be limited, conparisons between sexually abused males and females remain difficult. Refining current methodogies will help to clarify the differences between male and female CSA prevalence the and to effectively meet the needs of the abuse victims.

The type con Dild abuse texts, males do report more frecting betrayal than females, suggesting that differences in reporting rates for males reporting.
In fact, more adult males than females reported witnessing someone being killed, committing suicide, or being severely injured as a child, in addition to being severely physical attacked in childhood (Goldberg \& Freyd, 2006). Additionally, where partner and child abuse co-occur, males appear to be at elevated risk of physical abuse relative to females (Ross, 1996). Male children also reported experiencing more frequent and severe physical abuse in families with more extreme battering of the mothers, based on both child and mother reports (e.g., Jouriles \& Norwood, 1995). Teasing apart these nuanced interactions between abuse and gender will not only provide more comprehensive information about the context in which abuse occurs but also help to inform more effective prevention programs.

In addition to gender, sexual identity/orientation also appears to moderate risk of child abuse. A small number of studies using community samples found that individuals identifying as lesbian, gay, or bisexual (LGB) reported higher prevalence of physical abuse by family members as well as sexual abuse by family and nonfamily members during childhood and adolescence compared with individuals identifying as heterosexual (e.g., Saewyc et al., 2006). Children and adolescents who exhibit opposite-sex-linked behaviors also reported experiencing greater child abuse and neglect (McConaghy \& Silove, 1992); this may be due to the stigma associated with minority sexual orientation (Saewyc et al., 2006). Although earlier researchers had sometimes considered sexual and physical abuse to be a cause of LGB orientation, subsequent research has clearly contradicted this hypothesis. The majority of teens who identify as LGB do not report any abuse, and many teens who report sexual or physical abuse identify as heterosexual (e.g., Saewyc et al., 2006). Some studies suggest that adults identifying as bisexual appeared to be at higher risk of victimization in childhood and adolescence than gay and lesbian peers, thoutgh this finding has been inconsistent (see Saewyc et al., 2006). In the face of limited research to date, the mechanism underlying the additional risk for LGB individuals remains unclear. Some have suggested that stigma resulting from gender orientation decreases family protection and support for LGB teens (see Saewyc et al., 2006); however, many other potential links have yet to receive adequate research attention. In addition, researchers have yet to characterize the risk of child abuse faced by transgendered individuals, leaving gender identity sorely understudied in this field.

Age also moderates exposure risk. Specifically, younger children appear to be at elevated risk of some types of abuse and for severity of injuries. Nationwide data from the National Child Abuse and Neglect Data Systems (NCANDS) report that children ages 3 and younger are the most frequent victims of child fatality as a result of all types of child abuse and neglect (USDHHS, 2008). In the same nationally based data set, the overall rate of all types of child abuse and neglect was negatively correlated to the child's age. 
these findings are based on reported cases of abuse and neglect, which . may more strongly reflect trends in reporting rates than actual incidence rational

In contrast, when examining CSA of children under the Statistics, 2000). rates of reported CSA increase with age (Bureau of Justice Statilar correlations Research studies using adult retrospective reports also find similar correlations. bexual abuse rates and older age (e.g., Finkelhor, 1993; Putnam, 2003). Some rearchers also suggest that age as a risk factor for CSA operates differentially for girls than for boys, with risk starting earlier and lasting longer for . ir (Put 2003). In the context of betrayal trauma theory, girts may be girls (Put by a caregiver, whereas boys may be more likely more likely to be victimized buch as a priest, camp counselor, or coach to be victimized by a noncare (DePrince \& Freyd, 2002; Goldberg \& Freyd, 2006). Caregiver as perpe 作 the to the extent that the relationship between perpetrator and victim of function of the victim's gender, the age of onset and duration of also differ on the basis of gender.

Children with special needs may face an elevated risk of child abuse. Child protective services, The NCANDS repotte likely to be those with mental or physical disabilities or neglect than children without exposed to sexual abuse, physical abuse, or neglect than ching hospital files, disabilities (USDHHS, 2008). A series of studical and general population welfare records, and school reports in both clinical and general population samples found greater rates of malteat disabilities (for a review, types of disabilities compared with children withoutdion Sullivan \& Knutson, 2000). Additional studie ee Sullivan $\&$ Knite ship between specific disabilities and maltreatment types. with behavioral disorders have been found to be at haltreatment across all maltreatment; neglect was the most common form of mamerican Academy of types of disabilities (as reviewed in Hibbard, Desch, American Academy Pediatrics Committee on Child Abuse and of Pediatrics Council on Children with Disabilities, 2007). Resed risk of child of concluded that che type of maltreatment varies depend abuse and neglect, although the type of . Researchers also caution that thus far specific disability (Hibbard et al., 200 ). Research whether disabilities are a risk available findings do not allow us to determine whether disumetory theory for an outcome of, abuse. The developmental traumatology theory child's development will lead also suggests that trauna that occurs in cognitive and psychosial domain to psychopathology and impairments in cognitive ans the risk of additiona (De Bellis, 2001). In turn, these conditions increase the risk of additione some support abuse and neglect later in life. Data from various studis pr maltreatment at the for these hypotheses, such that disabilities increase risk of maltreatment at the
(Hibbard et al., 2007). Teasing apart directionality may be further complicated by recurrence rates, since the NCANDS also documented that child victims with a disability were $52 \%$ more likely to experience additional victimization than child victims without a disability (USDHHS, 2008).

Studies have repeatedly shown that violence itself begets violence. Once a child is exposed to abuse, he or she is at increased risk of exposure to additional incidents. According to the NCANDS data (USDHHS, 2008), children who incidents whe had not substantiated event of Additionally, studies have indicated that when a child . has experienced one type of maltreatment, the liketiho treatment co-occurring is very high (e.g., Finkelhor, Ormrod, \& Turner, 2007).

While we have so far primarily discussed gender, sexual identity/ orientation, age, and disabilities as some of the documented individual characteristics that place children at elevated risk of abuse, other studies have also documented additional vulnerability factors at the individual level (e.g., dhe commonality between all of these characteristics may point to important underlying vulnerabilities such as children's dependency, small size, and inability to defend themselves.

\section{Parental and Familial Context}

Rogosch and Cicchetti (2004) defined child maltreatment as parenting filures in acts of omission (i.e., neglect) or commission (i.e., abuse). Develfailures in acts of omission theory also views child abuse and neglect as extreme forms of dysfunctional family and interpersonal functioning (De Bellis, 2001). A forms of dystinerabilities in parental Accordingly, numers that increase risk of child maltreatment. Many researchers and familial contexts that increase risk of child maltreatment. Many researchers focus on parenting as a causal link between individual child characteristics (e.g., disabilities, young age) and maltreatment. We discuss some of the parenta and familial characteristics-including abuse history, parenting practices, substance abuse, co-occurring IPV, and family composition and interactionas contexts that place children at risk for abuse and as potential mechanisms of maltreatment.

A commonly held misconception has been that parents who were abused in childhood will necessarily continue the cycle of violence. Researchers have since documented a more complicated picture. Kaufman and Zigler's (1987) review found that only $30 \% \pm 5 \%$ of parents with a childhood abuse history go on to abuse their children. Thus, although not all parents who have experience maltreatment will abuse their children, childhood abuse history remains a risk factor for parental abuse of their children. 
Many parental factors have been associated with increased likelihood of . For example, young parental age, single parenthood, low education, abuse. For example, young parental age, single pall been found to low employment status, and parental psycho in Oliver, Kuhns, \& Pomeranz, be associated with physical abuse (as reviewed in 2006). However, are there common factors underlying the relation hips the increased risk of physical abuse as well as additional types of that drive the ind a social information pro maltreatment and which negative parenting beliefs (e.g., corporal punishment) fo child rearing (e.g., inability and maladaptive information processing related to child rearing regard to child to integrate situational cues, unrealistic ex physical child abuse. A review of compliance) are mothers who are physically abusive with mothers who are inctive behaviors and internal and stable attributions about their cheir children's positive behaviors external and unstable attributions about their children's posive be than nonasive mothers (Black et al., 2001). Abusive mothers were asoless likely to blame themselves for failed child-parent interactions than nonabusive mothers (Black et al., 2001). When considered within this context, the assomother (Black ciated matemal factors listed above may exacer macessing. A recent meta beliefs, attributions, or maladaptive information processing. A recent neglect analysis examining potential risk factors for child physink between parents also supports this pattern, finding a large effect size for the 4 . Stith et al. 2009). perceiving the child as a problem and child neglect $(r=.41$; Stith et a, 2009$)$. pello assimilation between these associated areas Although empirical studies on to pay more of research are yet to be conducted, researchers are starnal with additional attention to theories that integrate

factors (see Seng \& Prinz, 2008).

Research also indicates links between parental substance abuse and chational maltreatment (see Young, Boles, \& Otero, 2007). An estimate by 13.3 children Committee for Prevention of Child Abuse suggests that one in 13.3 children living with a substance-abusing parent experiences severe maltreag). Yet, what year (as cited in Besinger, Garland, Litrownik, \& Landsverk, 199). Yet, whect? year (as citedin Bubstance use and maltreatment or neglect? Research suggests that parents of drug-exposed infants may lack the necessary skills or resources to sufficiently deal with their infants' special needs (as reve may in Dore, Doris, \& Wright, 1995). Parents with ongoing substance use may in Dore, Doris, $\&$ Wrght spend a significant amount of time engaging in substable time spent on (e.g., acquiring, using), which reduces the amount neglect or failure to protect parenting, in turn increasing the likelihood of neglect or fille child. Further parin from maltreatment by others who have access to the child. Further, children from malith ffects of substance use may interfere with responsive parents coping with effects of substance ustress, again putting their children 62 CHU ET AL at risk of maltreatment. Future research needs to further examine these underlying mechanisms associated with substance abuse and risk of child maltreatment.

In many studies, parental stress emerges as a common - and potentially important-third variable. Stith et al.'s (2009) meta-analysis found a significant link between parents' level of stress and child neglect (effect size; $r=.38$ ). Under conditions of high stress, parents may be more likely to engage in rapid, automatic information processing (vs. more controlled flexible processing), which may increase the influence of basic belief structures on parenting behavior (Milner, 2000). Even beyond the main effect of stress on child abuse potential, researchers are discovering specific interactions between stress and additional life factors that contribute differentially to risk of child maltreatment. For example, the association between stress and physical child abuse potential was moderated by parents' belief in the value of corporal punishment (Milner, 2000). Specifically, among parents who strongly believed in the value of corporal punishment, level of stress was positively associated with physical child abuse potential. In contrast, level of parenting stress was not associated with physical child abuse potential among parents who reported low levels of belief in the value of corporal punishment. Looking specifically at substance abuse and parenting, Orme and Rimmer (1981) argued that outside factors, such as poverty or stress, may actually account for both substance use and the abusive behavior by caregivers. Delineating the impact of poverty and parental stress on child abuse risk is critically important to creating prevention programs. The authors argued that socioeconomic status complicates the relationship between substance abuse and child abuse because the poor and members of minority groups are overrepresented in hospital and treatment agency samples.

Family interactions and composition have also received wide attention. Findings document that partner abuse and child abuse frequently co-occur (for a review, see Herrenkohl, Sousa, Tajima, Herrenkohl, \& Moylan, 2008). Although witnessing violence between parents may be considered maltreatment in and of itself (or at least potentially traumatic), literature reviews point to high co-occurrence rates $(30 \%-60 \%)$ of documented child maltreatment and IPV (Edleson, 1999). Several factors are associated with the co-occurrence of child abuse and IPV, including lower socioeconomic status, lower parental education levels, unemployment or lower occupational status, substance use in the family, mental illness in caregivers, parenting stress, greater number of children or larger household size, and neighborhood violence (Herrenkohl et al., 2008). One hypothesized mechanism for the link between IPV and child abuse has focused on the perpetrator, if the aggression originates from the same individual. Alternatively, contextual factors, such as poverty and stress, may underlie the co-occurrence of partner and child abuse. Research has

(6) 
emerged to support this latter argument. For example, Margolin and Gordis (2003) found that parenting stress moderated the relationship between IPV aggression and child abuse potential such that the correlation was present in the context of high stress but not in the context of low stress. However, additional studies are needed to better understand stress as a mechanism of both IPV and maltreatment.

In related research, a large national survey of adolescents found that single-parent families reported more victimizations (from both strangers and nonstrangers) than two-parent families, even while controlling for race and socioeconomic status (Lauritsen, 2003). Various research studies as well as analyses of the NCANDS also indicate that youths living in blended households with a nonrelated parental figure are at higher risk of sexual and physical abuse as compared with youths living with two biological parents (see McRee, 2008). Additionally, Stith et al.'s (2009) meta-analysis found large effect sizes for the links between family conflict $(r=.39)$ and family cohesion $(r=-.32)$ to child physical abuse as well as parent-child relationship $(r=-.48)$ to child neglect. These associated factors help to give a more comprehensive understanding of the type of family composition and interactions that might exert additional influence on lower microsystem (i.e., individual-based) risk factors associated with child maltreatment.

\section{Environment and Social Network Factors}

Parenting does not occur in isolation but within a wider context of community and societal relationships. Thus, a discussion of risk and protective factors associated with child abuse must necessarily include a broader ecological perspective that considers environment and social factors. We review some of the major ecological correlates (e.g., poverty, neighborhood structure, community social support, religion, cultural identity) found to contribute to higher risk of child maltreatment. We also attempt to identify areas in which more specific mechanisms might drive these broad relationships or whether the larger environmental associations influence lower level processes that together contribute to child maltreatment.

Poverty-related factors such as low income level, median residential housing/property value, unemployment, and low education have been linked to increased risk of child abuse and neglect (Coulton. Crampton, Irwin, Spilsbury, \& Korbin, 2007; Ertem, Leventhal, \& Dobbs, 2000). Several structural characteristics of neighborhoods that go hand-in-hand with poverty are also associated with increased child abuse risk. For example, substandard or vacant housing, overcrowding, and residential instability are also linked to child abuse risk (as reviewed in Coulton et al., 2007). Child maltreatment rates in neighborhoods also have been shown to correlate with other indicators of social distress, such as infant mortality juvenile delinquency, violent crime, and drug trafficking (Coulton, Korbin, Su, \& Chow, 1995).

Although many links between poverty-related factors and abuse have been documented, we must be cautious in interpreting these findings. First, we do not yet know the processes by which social and economic characteristics of neighborhoods relate to child maltreatment. One possibility is that geographic concentrations of social disorganization (within which child abuse is more likely to occur) result from families already possessing multiple maltreatment risk factors forced to live in poor and unstable neighborhoods (Coulton et al., 2007). Furthermore, when child protective abuse rates are used as the primary data source, it remains unclear whether more abuse actually occurs in low-income homes or whether the increased involvement of system-based representatives is more likely to result in reports of abuse in low-income families relative to more well-to-do families where abuse also occurs. For example, research studies based on community surveys find almost no differences in CSA prevalence among socioeconomic classes, but a disproportionate number of CSA cases reported to child protective services come from families with lower socioeconomic'status (Finkelhor, 1993). Some researchers have suggested that professionals may feel more comfortable and confident labeling abuse among disadvantaged families, which tend to fit prevailing stereotypes about where abuse occurs (see Finkelhor, 1993). Alternatively, neighborhood chaos and social disruptions may directly influence maladaptive parenting practices, thus increasing abuse. For example, parents dealing with poverty-related stress may also be facing higher parenting stress, which leads to higher likelihood of maltreating their children (Coulton et al., 2007). Similar to the idea that within-level factors may influence each other (e.g., parental factors such as substance use and psychopathology affecting parenting practices), factors may also interact across levels (environmental context affecting parenting practices).

Neighborhood characteristics may also influence social relationships within the community, which in turn have an impact on maltreatment risk. For example, social support appears to be an important protective factor. Hunter and Kilstrom (1979) observed that parents who did not repeat child maltreatment were more likely to rely on a broad network of resources and social support, such as a positive relationship with one parent. Studies that have compared different communities based on interviews of residents, community leaders, and social service agency clients found that neighborhoods with low rates of child maltreatment reported greater social resources and community social network (as reviewed in Coulton et al., 2007). In contrast, Hashima and Amato (1994) found that the effect of poverty on punitive and unsupportive parenting behaviors was greater for parents reporting low social support.

To the extent that social support is an important protective factor, religious communities may offer parents (and children) important sources of 
support that protect against abuse. Religious groups also play an active and positive role in providing prevention and treatment programs for abuse victims. Parents engaging in abusive and/or neglectful behaviors tend not to be involved in community or religious activities (e.g., Polansky, Gaudin, Ammons, \& Davis, 1985). However, religious beliefs can encourage and justify violent, physically abusive parenting practices (Bottoms, Nielsen, Murray, \& Filipas, 2003). Thus, physical abuse may be perpetrated by adults who believe they are helping to deliver their children from sin. Taken together, religion appears to play a complex role in child abuse risk; currently, literature that identifies exact pathways in which religiosity and belief systems influence parental risk of perpetrating or rationalizing abuse remains limited.

Cultural identity also moderates abuse risk in complicated ways. Elliott and Urquiza (2006) reviewed studies examining cultural and ethnic differences in childhood maltreatment, specifically separating sexual abuse, physical abuse, and neglect. They concluded that findings across studies did not provide conclusive evidence for cultural group differences in CSA, with the exception of Asian cultures in which lower rates of CSA are reported. A similar picture emerged with physical abuse, where findings suggested various group differences, although the patterns remain inconsistent and at times contradictory. The authors attributed the inconsistency in physical abuse findings to methodological differences across studies and large within-culture and within-ethnicity variability. Although neglect was the most common form of child maltreatment according to the NCANDS (USDHHS, 2008), Elliott and Urquiza argued that the lack of studies focusing on cultural differences in neglect limits any conclusions.

Beyond pointing out cultural differences in prevalence rates, Elliott and Urquiza (2006) also reviewed cultural differences in potential mechanism that might explain the differing child abuse rates. For example, researchers have long suggested that how one interprets differences in reports of sexua abuse by cultural groups should be informed by ethnic differences in willing ness to disclose abuse as influenced by emphases on family and collectivistic beliefs, filial piety, and restraint in emotional expression. Researchers have also suggested that conservative norms in Asian cultures that frown on sexual activity act as a protective factor against CSA. Here again, reviews of studies provided no conclusive evidence regarding cultural/ethnic group differences in disclosure rates (Elliott \& Urquiza, 2006). Cultural attitudes and perceptions toward sexual activities and definitions of sexual abuse remain important areas of study to further investigate cultural patterns in risk for CSA.

For physical abuse, many studies have investigated differences in parenting among caregivers of different races and ethnicities, such as differences in disciplinary strategies, expression of warmth and affection, parenting attribu- tions, and definitions of what constitutes abuse. Differences in parenting appear to exist between cultural/ethnic groups; however, the differences also appear be influenced by a variety of factors, including acculturation levels, socioeconomic status, and neighborhood variables. Thus, any consideration of cultural identity in maltreatment, and in particular physical abuse, necessarily depends on understanding other familial, parental, and environmental factors.

Despite the inconsistency in cultural/ethnic differences in child maltreatment based on research studies, clear group differences exist in reported cases to child protective services. In 2002, African American, American Indian or Alaska Native, and multiracial children were the groups with the highest rates of maltreatment, with $19.8,15.9$, and 15.4 per 1,000 children of the same race or ethnicity, respectively (USDHHS, 2008). The next group consisted of White and Hispanic children (10.7 and 10.8 per 1,000 children of the same race or ethnicity). Asian children had the lowest victimization rate of 2.5 per 1,000 Asian children. Factors such as poverty may lead to heightened attention from state authorities and thereby disproportionately increase the number of cases reported among certain ethnic groups. Although research studies do point to variations in the child protective system's reporting of and response to reported child abuse from different cultural/ethnic backgrounds (Elliott \& Urquiza, 2006), societal and familial factors (e.g., exposure to community violence, single-parent homes) continue to confound the issue. Therefore, whether ethnic differences in prevalence rates in childhood are due to actual higher incidence of abuse or reporting rates remains unclear.

\section{HOW DO WE KNOW IT? THINKING CRITICÁLLY ABOUT METHODS, SAMPLES, AND ANALYSES}

Throughout our review of methodological factors (e.g., samples, analyses), we offered examples that should inform interpretation of findings. Here we summarize and highlight a few central methodological issues in this literature. For example, recruitment methods have played an important role in defining what we know about vulnerability and protective factors, with pros and cons to all of the methods used. Samples recruited through child protective services give us access to reports by an outside arbiter (e.g., a caseworker) who reports on the abuse, which may help with parent and child reporting biases that may be particularly likely to lead to underreporting among some cultural groups. However, reliance on substantiated or child protective samples will likely lead us to find stronger associations between risk and poverty-related factor than in other sampling methods. Families living in poverty have more contact with system-based representatives and may, therefore, be more likely to have 
potentially abusive or neglectful behaviors reported to the authorities than wealthier families. Of course, many of the conditions of poverty do create terrible risks for children, such as unstable living arrangements, lack of parental education, and poor access to medical care. The interesting methodological questions become how to both understand the particular risk created by poverty and access higher socioeconomic samples outside the system where risk factors may differ.

The most common alternatives to child protective samples are parent reports or adult retrospective reports. Parent reports of abuse to researchers, including those who are mandated reporters, may increase the risk of false negatives. Adult retrospective reports of abuse may also be vulnerable to underreporting (particularly of sexual traumas by trusted others) because of high rates of false negatives (Bolen \& Scannapieco, 1999). However, retro spective reports of abuse have some pronounced advantages over documente samples. Kendall-Tackett and Becker-Blease (2004) identified at least two major advantages of retrospective reports over documented samples: (a) The are less prone to missed cases and underreporting (as so few abuse cases ge documented), and (b) they are more representative of most cases of abuse in that documenting abuse requires numerous factors and has pronounced impact on the victim).

The majority of studies reviewed here involved cross-sectional, retrospective methods (i.e., the abuse already occurred). By looking primarily at samples in which abuse has already occurred, we bias our research towar identifying risk factors. Identifying risk factors is important and valuable for prevention; however, finding methods to study protective factors that kept children safe from abuse remains a challenge. To date, the majority of studies examining protective factors have focused on protective factors as part of resiliency after exposure to trauma. All too often, in studies that examine contributors to child abuse and neglect, we found that protective factors were simply the flip side of vulnerability factors identified in crosssection research. That is, if female gender is a risk factor for one type of abuse, then male gender is protective. Only a few studies identified protective factors. In these cases, traits provided buffers for parents already facing adversity and risk for engaging in abuse. For example, parental coping and social support seemed to protect against child physical abuse (see Black et al., 2001; Coulton et al., 2007). However, parental and environmental factors that may protect against CSA and neglect remain unclear. Therefore, at this time, we know little about protective factors independent of risk. Further, the cross-sectional nature of this research also means that we know little about how risk factors change over time. This is particularly important considering the tremendous cognitive, emotional, and social development that occurs in the first 18 years of life.

CHU ET AL.

\section{WHERE DO WE GO FROM HERE?}

The translation of research findings into action in the form of parent education, policy changes, or prevention programs remains an important step. In this section, we present recommendations for future research and policy that may bridge some of the gaps in the current state of knowledge on and services for victims of child abuse.

\section{Next Research Steps}

Research in this field began by documenting broad group differences (e.g., gender, culture/ethnicity) and correlations (e.g., poverty, stress) associated with maltreatment rates and child maltreatment. As many researchers (e.g., Cicchetti \& Lynch, 1993; Coulton et al., 2007) have suggested, we must start to use a more ecological perspective that includes all levels of analysis. These third-generation studies using more complex analyses and multiple methods will help us more fully understand the context within which child abuse happens (e.g., Banyard \& Williams, 2007). Such studies may clarify nonlinear processes of risk and protective factors of child abuse, which in turn can further inform risk for further victimization and how maltreatment may unfold across the life span. Such research also has important implications for clinical practice with survivors of child maltreatment as it builds an empirical base of knowledge to identify resources for interventions that are empowering and build on survivors' strengths.

Because children change greatly during the course of development from infancy to adolescence, examining child victimization within a developmental perspective will also shed further light on changes in individual and environmental risk factors over time. According to the developmental psychopathology and developmental traumatology perspectives, researchers need to be mindful of how trauma might disrupt normal development in multiple domains of functioning as well as different physiological systems (Cicchetti \& Toth, 2005; De Bellis, 2001). Characteristics that place children at risk of certain types of maltreatment may vary as a function of their developmental stage. Risk of exposure to different types of maltreatment may change as children assert greater independence from caregivers. The trauma exposure may also differentially impact various areas of functioning, depending on the timing of the developmental process. Further research is needed to minimize risk for children at all stages of development.

In addition, relatively little attention has been paid to within-group differences and even less attention paid to subpopulations (e.g., Pacific Islanders, Filipino Americans, Cuban Americans, Alaskan Natives). Studies that focus on differences between broadly defined groups ignore potentially important 
intragroup differences and obscure true cultural differences that affect prevalence, reporting, and experiences of child maltreatment. For example, third generation Chinese Americans and recently immigrated Hmong refugees are both categorized as "Asian American." However, the manner in which Hmong family perceives and addresses child maltreatment, the family's risk of engaging in child abuse, and parenting attitudes may differ widely from those of a third-generation Chinese American family (Elliott \& Urquiza, 2006).

\section{Recommendations for Policy}

The studies reviewed here point to several important policy issues related to child welfare practices. For example, continued training is needed for professionals who interact with children and families across diverse settings (e.g., medical system) to recognize and assess maltreatment based on cuttingedge research on vulnerability and protective factors. Researchers such as Hibbard et al. (2007) have noted that many health workers do not receive sufficient training on the identification of risk factors, such as disabilities, for child abuse and neglect.

Because the literature on vulnerability and protective factors has been anything but static, trainings must be updated as research continues to identify vulnerability and protective factors as well as complex interactions among those factors. Training professionals to identify children who are at risk of abuse or who have been abused must, therefore, capture the complex relationship between risk factors. For example, it would be an unwelcome outcome if data on ethnicity and abuse led to focused attention on certain subgroups without considering how interactions between ethnic identity and other vulnerability protective factors contribute to risk across and within groups. With up-to-date training, health care providers and other professionals will be in a unique position to use their knowledge to identify maltreated children as well as educate parents and families on ways to protect children. Health care professionals urgently need to learn to better use this body of research for risk assessment to prevent maltreatment from occurring, which will have to involve both policy and research agendas. For example, rigorous research methods are needed to examine whether the vulnerability and protective factors identified in documented abuse reflect actual underlying differences (e.g., between groups) versus biases in the system.

Additionally, prevention of maltreatment requires programs to systematically and comprehensively address multiple risk and protective factors, including the family and community (Herrenkohl et al., 2008). While we recommend that future research target multiple levels of influence on maltreatment, so too should policymakers address the implications of multiple system interactions. For example, mothers who are victimized by their partners

CHU ET AL. may not be able to fully protect their children because leaving the abusive partner may result in loss of their economic support or increased safety risks to mothers and children. While ensuring the safety of children is paramount, policies can help to strengthen communities to support families and to reduce prevalence of child maltreatment (Coulton et al., 2007). Community social support appears to be an especially important protective factor that warrants further research to identify specific policies that can build this capacity to decrease child abuse risk.

A more collaborative team-approach response will help future data "collection and provide more comprehensive responses to children who have experienced maltreatment. For example, because there is no federal mandate that information be collected on families involved in multiple systems (e.g., a family with identified child abuse that is also engaged in mandated substance treatment), the information available on how risk and protective factors interact is largely an estimate based on an interpolation of research studies. Future studies should establish accurate estimates of families who access multiple systems to more fully assess the need for services, and in turn develop appropriate capacity to respond to that need. In particular, states and communities need to fully understand the size, scope, and extent of the issue of families who are involved in systems in their jurisdictions because these factors may vary depending on geographic locations and neighborhood characteristics. Finally, we note the need for a substantial increase in federal funding for child abuse research. Freyd et al. (2005) proposed the creation of a new National Institute of Child Abuse and Neglect to help accomplish this goal. Such a bold move is justified by the economic, health, and human impact of these problems.

\section{SUMMARY AND CONCLUSIONS}

On the basis of findings from research studies and national statistics of reported child maltreatment cases, we have evidence that certain groups of children are at higher risk of maltreatment compared with their peers. These risk factors may be generally categorized as occurring at the individual, parental and familial, and environmental and societal levels. This body of research clearly illustrates that child maltreatment cannot be reduced to a single-risk model. Instead, factors across and within systems influence each other in additive and interactive ways to increase or decrease potential for child abuse and neglect. Importantly, even while we continue to gain a better understanding of the interaction between risk factors, many of the mechanisms that underlie these interactions remain poorly understood. With the important advances in the trauma field, future research studies with more nuanced research questions and sophisticated study designs and methodology will help us to

VULNERABILITY AND PROTECTIVE FACTORS 
address these unanswered questions. In turn, researchers and policymakers must work together to provide comprehensive and collaborative services in both the prevention and treatment of child maltreatment. To date, some prevention programs have attempted to educate parents on caretaking behaviors, attitudes, and beliefs in an effort to enhance protection against risk for abuse and neglect of their children. Researchers have also established programs to shore up social support networks within high-risk communities in efforts to prevent child abuse and neglect in those neighborhoods. However, the effectiveness of these prevention programs remains unclear. Thus, we also need to continue to monitor how to most effectively translate advances in research into prevention of abuse and neglect through policies and treatment programs.

\section{REFERENCES}

Banyard, V., \& Williams, L. (2007). Women's voices on recovery: A multi-method study of the complexity of recovery from child sexual abuse. Child Abuse E'Neglect, 31, 275-290. doi: $10.1016 /$ j.chiabu.2006.02.016

Besinger, B. A., Garland, A. F., Litrownik, A. J., \& Landsverk, J. A. (1999). Caregiver substance abuse among maltreated children placed in out-of-home care. Child Welfare Joumal, 78, 221-239.

Black, D. A., Heyman, R. E., \& Slep, A. M. S. (2001). Risk factors for child physical abuse. Aggression and Violent Behavior, 6, 121-188. doi:10.1016/S1359-1789(00) $00021-5$

Bolen, R. M., \& Scannapieco, M. (1999). Prevalence of child sexual abuse: A corrective meta-analysis. Social Service Review, 73, 281-313. doi:10.1086/514425

Bottoms, B. L., Nielsen, M., Murray, R., \& Filipas, H. (2003). Religion-related child physical abuse: Characteristics and psychological outcomes. Joumal of Aggression, Maltreatment \& Trauma, 8, 87-114.

Bureau of Justice Statistics. (2000). National crime victimization survey. Washington, DC: U.S. Department of Justice.

Cicchetti, D., \& Lynch, M. (1993). Toward an ecological/transactional model of community violence and child maltreatment: Consequences for children's development. Psychiatry, 56, 96-118.

Cicchetti, D., \& Toth, S. L. (2005). Child maltreatment. Annual Review of Clinical Psychology, 1, 409-438. doi:10.1146/annurev.clinpsy.1.102803.144029

Coulton, C. J., Crampton, D. S., Irwin, M., Spilsbury, J. C., \& Korbin, J. E. (2007). A review of the literature and alternative pathways. Child Abuse E Neglect, 31 , 1117-1142. doi:10.1016/j.chiabu.2007.03.023

Coulton, C. J., Korbin, J. E., Su, M., \& Chow, J. (1995). Community level factors and child maltreatment rates. Child Development, 66, 1262-1276. doi:10.2307| 1131646
DePrince, A. P., \& Freyd, J. J. (2002). The intersection of gender and betrayal trauma. In R. Kimerling, P. C. Oumette, \& J. Wolfe (Eds.), Gender and PTSD (pp. 98-113). New York, NY: Guilford Press.

De Bellis, M. D. (2001). Developmental traumatology: The psychobiological development of maltreated children and its implications for research, treatment, and policy. Development and Psychopathology, 13, 539-564. doi:10.1017/ S0954579401003078

Dore, M. M., Doris, J. M., \& Wright, P. (1995). Identifying substance abuse in maltreating families: A child welfare challenge. Child Abuse \& Neglect, 19, 531-543. doi: 10.1016/0145-2134(95)00013-X

Edinburgh, L., Saewyc, E. M., Thao, T., \& Levitt, C. (2006). Sexual exploitation of very young Hmong girls. Journal of Adolescent Health, 39, 111-118. doi:10.1016 j.jadohealth.2005.09.003

Edleson, J. L. (1999). Children's witnessing of adult domestic violence. Journal of Interpersonal Violence, 14, 839-870. doi:10.1177/088626099014008004

Elliott, K., \& Urquiza, A. (2006). Ethnicity, culture, and child maltreatment. Journal of Social Issues, 62, 787-809. doi:10.1111/j.1540-4560.2006.00487.x

Ertem, I. O., Leventhal, J. M., \& Dobbs, S. (2000). Intergenerational continuity of child physical abuse: how good is the evidence? The Lancet, 356, 814-819. doi: 10.1016/S0140-6736(00)02656-8

Finkelhor, D. (1993). Epidemiological factors in the clinical identification of child sexual abuse. Child Abuse \& Neglect, 17, 67-70. doi:10.1016/0145-2134 (93) 90009 -T

Finkelhor, D., Hotaling, G., Lewis, I. A., \& Smith, C. (1990). Sexual abuse in a national survey of adult men and women: Prevalence, characteristics, and risk factors. Child Abuse \& Neglect, 14, 19-28. doi:10.1016/0145-2134(90)90077-7

Finkelhor, D., Ormrod, R. K., \& Turner, H. A. (2007). Polyvictimization and trauma in a national longitudinal cohort. Development and Psychopathology, 19, 149-166.

Freyd, J. J., DePrince, A. P., \& Gleaves, D. (2007). The state of betrayal trauma theory: Reply to McNally - conceptualizations and future directions. Memory, 15, 295-311. doi:10.1080/09658210701256514

Freyd, J. J., Putnam, F. W., Lyon, T. D., Becker-Blease, K. A. Cheit, R. E., Siegel, N. B., \& Pezdek, K. (2005, April 22). The science of child sexual abuse. Science, 308 , 501. doi:10.1126/science.1108066

Goldberg, L., \& Freyd, J. J. (2006). Self-reports of potentially traumatic experiences in an adult community sample: Gender differences and test-retest stabilities of the items in a Brief Betrayal-Trauma Survey. Journal of Trauma \& Dissociation, 7(3), 39-63. doi:10.1300/J229v07n03_04

Hashima, P. Y., \& Amato, P. R. (1994). Poverty, social support, and parental behavior. Child Development, 65, 394-403. doi:10.2307/1131391

Herrenkohl, T. I., Sousa, C., Tajima, E. A., Herrenkohl, R. C., \& Moylan, C. A. (2008). Intersection of child abuse and children's exposure to domestic violence. Trauma, Violence \& Abuse, 9, 84-99. doi:10.1177/1524838008314797 
Hibbard, R. A., Desch, L. W., American Academy of Pediatrics Committee on Child Abuse and Neglect, \& American Academy of Pediatrics Council on Children With Disabilities. (2007). Maltreatment of children with disabilities. Pediatrics, 119, 1018-1025. doi:10.1542/peds.2007-0565

Holmes, W. C., \& Slap, G. B. (1998). Sexual abuse of boys: Definition, prevalence, correlates, sequelae, and management. JAMA, 280, 1855-1862. doi:10.1001/ jama.280.21.1855

Hunter, R. S., \& Kilstrom, N. (1979). Breaking the cycle in abusive families. The American Journal of Psychiatry, 136, 1320-1322.

Jouriles, E. N., \& Norwood, W. D. (1995). Physical aggression toward boys and girl in families characterized by the battering of women. Joumal of Family Psychology, 9, 69-78. doi:10.1037/0893-3200.9.1.69

Kaufman, J., \& Zigler, E. (1987). Do abused children become abusive parents? American Journal of Orthopsychiatry, 57, 186-192

Kendall-Tackett, K., \& Becker-Blease, K. A. (2004). The importance of retrospective findings in child maltreatment research. Child Abuse \& Neglect, 28, 723-727. doi:10.1016/j.chiabu.2004.02.002

Lauritsen, J. L. (2003). How families and communities influence youth victimization. Washington, DC: U.S. Department of Justice, Office of Justice Programs, Office of Juvenile Justice and Delinquency Prevention.

Margolin, G., \& Gordis, E. B. (2003). Co-occurrence between marital aggression and parents' child abuse potential: The impact of cumulative stress. Violence and Victims, 18, 243-258. doi:10.1891/vivi.2003.18.3.243

McConaghy, N., \& Silove, D. (1992). Do sex-linked behaviors in children influence relationships with their parents? Archives of Sexual Behavior, 21, 469-479. doi:10.1007/BF01542271

McRee, N. (2008). Child abuse in blended households: Reports from runaway and homeless youth. Child Abuse 82 Neglect, 32, 449-453.

Milner, J. S. (2000). Social information processing and child physical abuse: Theory and research. In D. J. Hansen (Ed.), Nebraska Symposium on Motivation: Vol. 46. Motivation and child maltreatment (pp. 39-84). Lincoln, NE: University of Nebraska Press.

Oliver, W. J., Kuhns, L. R., \& Pomeranz, E. S. (2006). Family structure and child abuse Clinical Pediatrics, 45, 111-118. doi:10.1177/000992280604500201

Orme, T. C., \& Rimmer, J. (1981). Alcoholism and child abuse: A review. Journal of Studies on Alcohol, 42,273-287.

Polansky, N. A., Gaudin, J. M., Ammons, P. W., \& Davis, K. B. (1985). The psychological ecology of the neglectful mother. Child Abuse \& Neglect, 9, 265-275. doi:10.1016/0145-2134(85)90019-5

Putnam, F. W. (2003). Ten-year research update review: Child sexual abuse. Joumal of the American Academy of Child and Adolescent Psychiatry, 42, 269-278. doi:10.1097) 00004583-200303000-00006
Rogosch, F. A., \& Cicchetti, D. (2004). Child maltreatment and emergent personality organization: Perspectives from the five-factor model. Journal of Abnormal Child Psychology, 32, 123-145. doi:10.1023/B:JACP.0000019766.47625.40

Romano, E., \& De Luca, R. V. (2001). Male sexual abuse: A review of effects, abuse characteristics, and links with later psychological functioning. Aggression and Violent Behavior, 6, 55-78. doi:10.1016/S1359-1789(99)00011-7

Ross, S. M. (1996). Risk of physical abuse to children of spouse abusing parents. Child Abuse \& Neglect, 20, 589-598. doi:10.1016/0145-2134(96)00046-4

Saewyc, E. M., Skay, C. L., Pettingell, S. L., Reis, E. A., Bearinger, L., Resnick, M., . . Combs, L. (2006). Hazards of stigma: The sexual and physical abuse of gay, lesbian, and bisexual adolescents in the United States and Canada. Child Welfare, 85 195-213.

Seng, A. C., \& Prinz, R. J. (2008). Parents who abuse: What are they thinking? Clinical Child and Family Psychology Review, 11, 163-175. doi:10.1007/s10567-008-0035-y

Senn, T. E., Carey, M. P., \& Vanable, P. A. (2008). Childhood and adolescent sexual abuse and subsequent sexual risk behavior: Evidence from controlled studies, methodological critique, and suggestions for research. Clinical Psychology Review, 28, 711-735. doi: 10.1016/j.cpr.2007.10.002

Stevens, I., \& Hassett, P. (2007). Applying complexity theory to risk in child protection practice. Childhood, 14, 128-144. doi:10.1177/0907568207072535

Stith, S. M., Liu, T., Davies, C., Boykin, E. L., Alder, M. C., Harris, J. M., . . . Dees, J. E. M. E. G. (2009). Risk factors in child maltreatment: A meta-analytic review of the literature. Aggression and Violent Behavior. 14, 13-29. doi:10.1016/j.avb. 2006.03.006

Sullivan, P. M., \& Knutson, J. F. (2000). Maltreatment and disabilities: A populationbased epidemiological study. Child Abuse \& Neglect, 24, 1257-1273. doi:10.1016 S0145-2134(00)00190-3

Tang, S. S., Freyd, J. J., \& Wang, M. (2008). What do we know about gender in the disclosure of child sexual abuse? Journal of Psychological Trauma, 6(4), 1-26. doi:10.1080/19322880802096442

U.S. Department of Health and Human Services. (2008). Child maltreatment 2006 Reports from the states to the National Child Abuse and Neglect Data System. Washington, DC: US Government Printing Office.

Young, N. K., Boles, S. M., \& Otero, C. (2007). Parental substance use disorders and child maltreatment: Overlap, gaps, and opportunities. Child Maltreatment, 12 37-149. dol:10.1177/1072559507300322 